\title{
Research Article \\ Effect of Catalyst Morphology on the Quality of CVD Grown Graphene
}

\author{
Ya-Ping Hsieh, ${ }^{1}$ Yi-Wen Wang, ${ }^{1}$ Chu-Chi Ting, ${ }^{1}$ Hsiang-Chen Wang, \\ Kuang-Yao Chen, ${ }^{2}$ and Chang-Chung Yang ${ }^{2}$ \\ ${ }^{1}$ Graduate Institute of Opto-Mechatronics, National Chung Cheng University, 168 University Road, Min-Hsiung, \\ Chiayi 62102, Taiwan \\ ${ }^{2}$ Green Energy and Environment Research Laboratories, Industrial Technology Research Institute, Hsinchu, Taiwan
}

Correspondence should be addressed to Ya-Ping Hsieh; yphsieh@ccu.edu.tw

Received 15 March 2013; Revised 9 July 2013; Accepted 17 July 2013

Academic Editor: Nadya Mason

Copyright (C) 2013 Ya-Ping Hsieh et al. This is an open access article distributed under the Creative Commons Attribution License, which permits unrestricted use, distribution, and reproduction in any medium, provided the original work is properly cited.

\begin{abstract}
The strong interest in graphene has motivated large effort in the scalable production of high-quality material. The potential of chemical vapor deposition on $\mathrm{Cu}$ foil to produce such graphene is impeded by lacking understanding of the relation between catalyst properties and graphene performance. We here present a systematic analysis of the catalyst morphology and its effect on electrical properties of graphene. We find that nanometer sized particles increase the density of bilayer regions but have no significant effect on carrier transport. Long wavelength roughness (waviness), on the other hand, generates defective graphitic regions that deteriorate carrier mobility. These findings shed light on the graphene formation process on $\mathrm{Cu}$ substrates and open a route to improve graphene quality for electronics applications.
\end{abstract}

\section{Introduction}

Graphene, a single atomic layer of carbon atoms, has generated enormous attention due to its physical properties. Proof-of-concept experiments demonstrated novel electronic and optoelectronic devices in transistors [1], solar cells [2, 3], photodetectors [4], and so forth. The desire for large-scale application of this material has motivated the development of a number of methods to synthesize large-area graphene sheets. Amongst these approaches, the chemical vapor deposition (CVD) synthesis of graphene on $\mathrm{Cu}$ substrate $[5,6]$ has shown great promise for producing high-quality singlelayer graphene. Despite significant efforts, the properties of CVD graphene have yet to reach the requirements of electronics applications for mobility and uniformity. Recent reports emphasize the importance of the surface morphology of the catalytic $\mathrm{Cu}$ substrate in determining the homogeneity and electronic transport properties of the grown graphene film [7-9]. It was found that imperfections in the Cu substrate interfere with graphene growth. Furthermore, commonly used $\mathrm{Cu}$ foil is usually covered with a layer of chromium oxide for anticorrosion protection [10] that can affect the properties of grown graphene. Consequently, the formation of smooth $\mathrm{Cu}$ surfaces free of contaminants becomes a necessary step for the synthesis of high-quality graphene.

Despite this importance of the catalyst pretreatment, little work has been done to correlate the condition of the $\mathrm{Cu}$ catalyst with the properties of the obtained graphene. We here present the first systematic study of the effect of catalyst morphology on the electrical and optical properties of graphene. We find that a higher density of surface particles supports the formation of bilayer graphene regions but has little effect on the electrical properties of the graphene film. Low frequency roughness (waviness), on the other hand, deteriorates the quality of graphene significantly as studied by Raman spectroscopy and electrical measurements.

These observations provide deeper understanding of the graphene growth and have large significance for the optimization of graphene quality for future applications. 


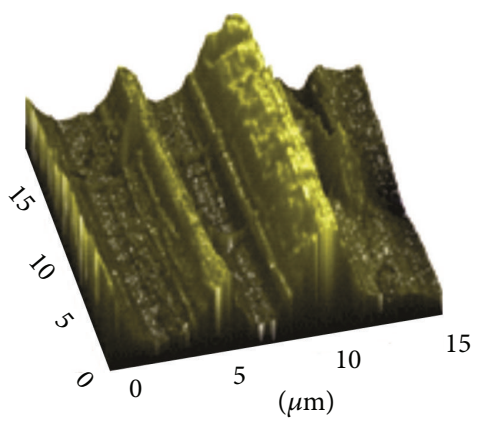

(a)

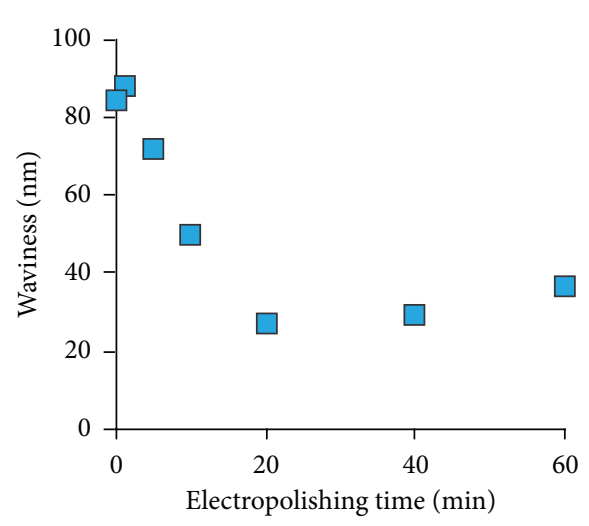

(d)

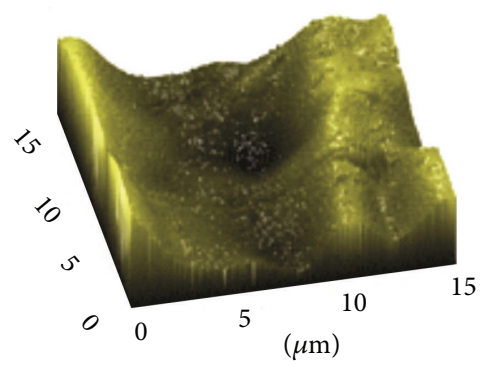

(b)

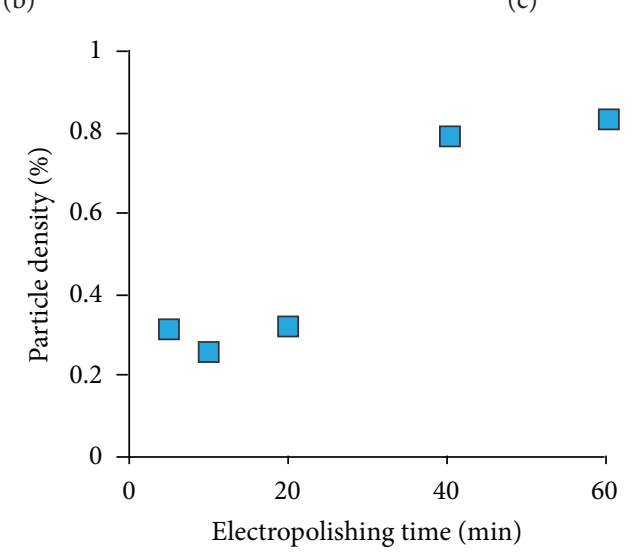

(e)

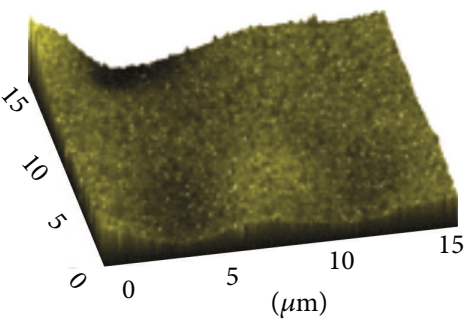

(c)

Figure 1: Change of Cu morphology upon electropolishing: (a)-(c) AFM images for 5 min (a), 10 mins (b), 40 mins (c) electropolishing, (d) waviness versus electropolishing time, and (e) particle density versus electropolishing time.

\section{Experimental}

Electrochemical polishing was employed to control the morphology of the $\mathrm{Cu}$ foil. First, copper foil (99.8\%, Alfa-Aesar, no. 13382) was precleaned by sonication in acetic acid for $5 \mathrm{~min}$ to remove the oxide layer. The dried $\mathrm{Cu}$ foil was then affixed on a glass slide to simplify handling. The $\mathrm{Cu}$ foil was placed into a breaker containing a solution of $300 \mathrm{~mL}$ of $\mathrm{H}_{3} \mathrm{PO}_{4}(85 \%)$ and $100 \mathrm{~mL}$ of polyethylene glycol (PEG, molecular weight 400, from Sigma-Aldrich Co.) as electrolytic solution. This $\mathrm{Cu}$ foil was then used as working electrode, and another $\mathrm{Cu}$ plate was used as counter electrode, and a DC power supply was used to apply a voltage of $1.5 \mathrm{~V}$. After the electropolishing treatment, the sample was washed with copious amounts of deionized water and then rinsed with isopropyl alcohol, followed by blow-drying with $\mathrm{N}_{2}$.

In our acid etching pretreatment, nitric acid (TFB, TRANSENE Company, Inc.) and 37\% HCl (J.T baker company) were used as obtained.

The pretreated $\mathrm{Cu}$ foils were then used to carry out graphene growth. For this, the reactor chamber was pumped down to 10 mTorr using a vacuum pump. Hydrogen gas was introduced into the chamber during heating to $1000^{\circ} \mathrm{C}$. The foils were annealed for $70 \mathrm{~min}$ to initiate $\mathrm{Cu}$ grain growth and to remove organic residue and surface oxide. Graphene growth was then performed at $1000^{\circ} \mathrm{C}$ for 100 min under a gas mixture of $\mathrm{H}_{2}(100 \mathrm{sccm})$ and $\mathrm{CH}_{4}(40 \mathrm{sccm})$. The sample was then rapidly cooled under 10 sccm hydrogen flow. Graphene samples were transferred by a PMMA assisted process in which $\mathrm{Cu}$ was etched away by $5 \% \mathrm{FeCl}_{3}$, and graphene was transferred onto $\mathrm{SiO}_{2} / \mathrm{Si}$ wafers. Finally, the PMMA was dissolved by immersion in acetone.

Carrier mobility was obtained by Hall effect measurements of macroscopic $(10 \times 10 \mathrm{~mm})$ films thus transferred employing a Van der Pauw geometry. Upon optimization of the transfer protocol, sample-to-sample variations were less than $10 \%$.

Gwyddion software was used to extract the waviness and particle density from AFM images. The waviness parameter was defined as the RMS roughness of a sample after high-pass filtering removed features with wavevectors larger than $k=$ $5 \mu \mathrm{m}^{-1}$.

A watershed algorithm was applied to identify particles and calculate their area densities.

\section{Results and Discussion}

In a first set of experiments we analyzed the effect of electropolishing duration on the morphology of the $\mathrm{Cu}$ foil. Atomic force microscope images reveal the change in roughness as time progresses (Figures $1(\mathrm{a})-1(\mathrm{c})$ ). The images reveal, however, that the roughness does not simply decrease as assumed in previous electropolishing papers. Instead, two 


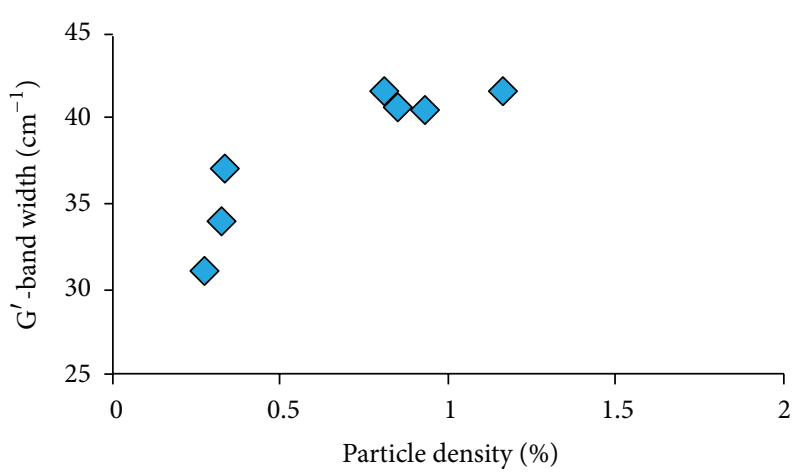

(a)

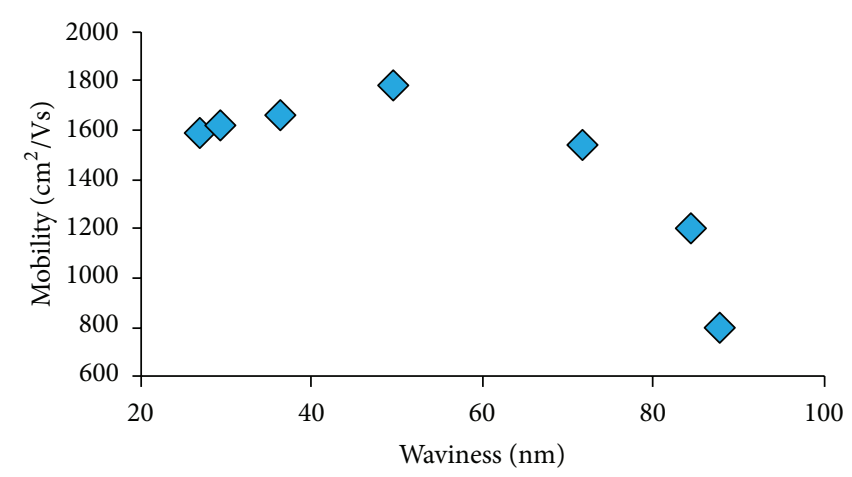

(b)

FIGURE 2: Graphene quality for different Cu morphology: (a) G' peak width versus particle density; (b) carrier Hall mobility versus waviness.

components of the roughness have to be distinguished: while the long range roughness (or waviness) decreases as a function of electropolishing time (Figure 1(d)), small particles start occurring (Figure 1(e)). This is thought to be caused by two different mechanisms taking place during electropolishing. The concentration of electric field around micrometer high protrusions causes preferential electrochemical etching of these features and consequently adecrease of the waviness [7]. Nanometer sized particles, however, exhibit too small field enhancement and are not affected by the same mechanism [11]. Consequently, protrusions will smooth out until they reach a critical size but will remain unchanged afterwards. Therefore, increasing electropolishing time represents a tradeoff between decreasing waviness and increasing particle density as shown when comparing Figures $1(\mathrm{~d})$ and $1(\mathrm{e})$.

Based on the finding that waviness and particle density are both changed during electropolishing, we investigate the effect of each of the two parameters on the quality of graphene synthesized from thus pretreated $\mathrm{Cu}$ foils.

Raman spectroscopy reveals an increase of the $G^{\prime}$ peak width as a function of particle density (Figure 2(a)). This behaviour is attributed to an increasing fraction of bilayer regions in the sample. The $G^{\prime}$ Raman feature originates from a double resonance process of two optical phonons at the K-point which is very sensitive to changes in the graphene band structure. A larger number of allowed electronic transitions in bilayer graphene broaden the $G^{\prime}$ band which agrees with our observation. The absence of a sharp transition between single-layer and bilayer graphene between individual Raman spectra indicates that even in samples with high particle densities, both bilayer and single-layer graphene can be found within a laser spot of $5 \mu \mathrm{m}$ diameter.

This observation agrees with previous reports that particles initiate growth of bilayer graphene due to local supersaturation by methane and subsequent nucleation of a second graphene layer [10]. These graphene layers, however, are small compared to the single-layer regions, and no effect of their presence on electrical transport was found.

This finding is significant, since several reports highlight the correlation of increased particle density with the formation of smaller graphene grains $[9,12]$. The lacking strong trend of graphene defectiveness or carrier mobility with particle density, however, does not support that conclusion. Instead, the observation confirms results by Tsen et al. [13] tailoring electrical transport across grain boundaries in polycrystalline graphene that intergrain carrier transport is not the limiting factor for the film mobility for well connected grains.

Surprisingly, however, we observe a strong correlation of the substrate waviness with the carrier mobility of the grown graphene (Figure 2(b)). It can be seen that beyond a critical value of $\sim 70 \mathrm{~nm}$, the carrier mobility decreases rapidly with waviness. This behaviour can be explained when examining the graphene growth process during CVD. Hydrocarbons are adsorbed on the $\mathrm{Cu}$ substrate and diffuse on the surface until they attach to the edge of an existing graphene grain or a step edge [14]. In the case of samples with larger waviness, more step edges occur. These features act as obstacles for high-quality graphene growth and instead form disordered graphitic regions. The presence of disordered graphene regions can have two effects. First, carrier transport through these regions proceeds less efficiently, and the overall mobility of the graphene film deteriorates. Even if the disordered material is not integrated in the graphene film but only positioned in contact with pristine graphene, it can produce additional carrier scattering through charged impurities, phonon coupling, or electrostatic interactions.

In order to confirm the detrimental effects of the waviness on the carrier transport, we chose a second approach to control the substrate waviness independently of the particle density. To achieve this waviness control, we etched copper foil in hydrochloric acid and commercial nickel etchant (TFB) for varying durations between 1 minute and 20 minutes. A clear increase in waviness can be seen for extended etching times (Figure 3(a)). No such trend on etching time was found for the particle density since isotropic erosion occurs in the acid etching process.

In agreement with the previous results shown in Figure 2(b), there is a strong effect of waviness on the carrier mobility (Figure 3(b)). Consequently, the sheet resistance, which is commonly used as a figure of merit, increases with waviness (Figure 3(c)). These observations support our previous hypothesis that an increasing roughness of the $\mathrm{Cu}$ 


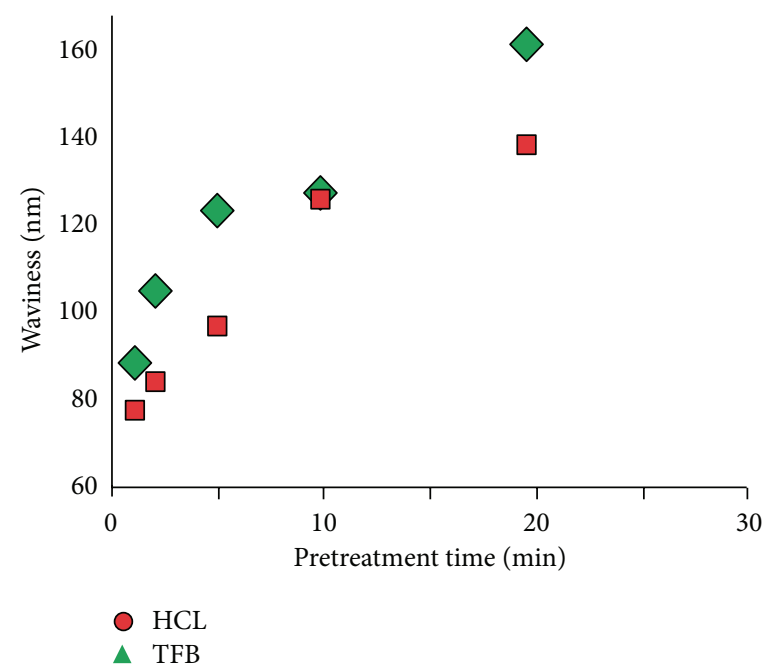

(a)

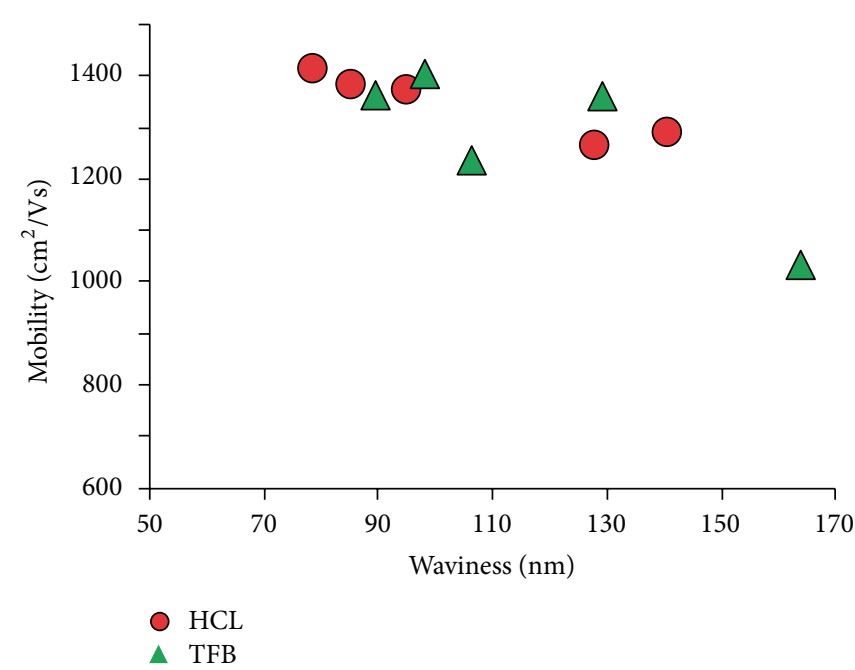

(b)

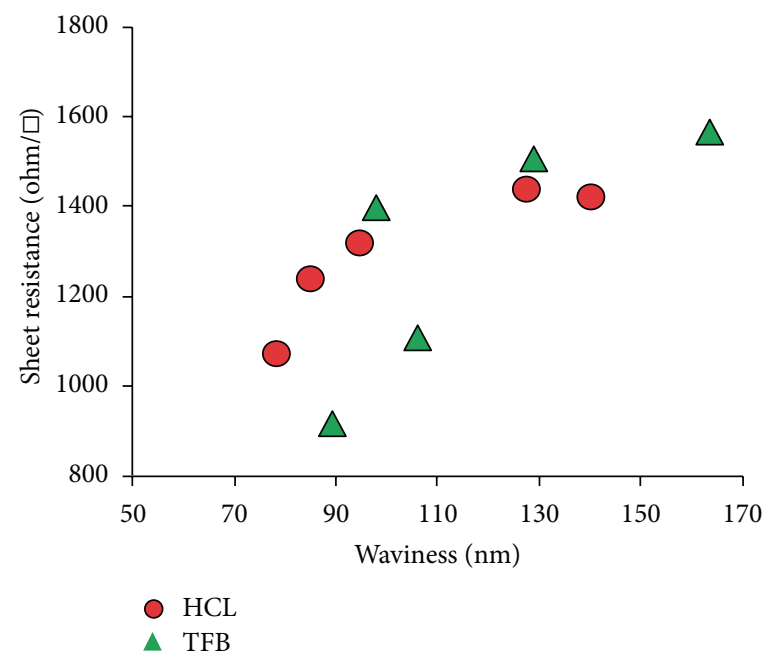

(c)

FIGURE 3: Effect of acid pretreatment conditions for 10 different graphene samples: (a) waviness versus etching time, (b) carrier Hall mobility versus waviness, and (c) film sheet resistance versus waviness.

substrate results in larger amounts of defective graphene which impedes carrier transport.

Raman spectroscopy was employed to further confirm this point. We find that longer etching increases the density of dark regions in the graphene film as seen when comparing Figures 4(a) and 4(b). The growth conditions for these two samples were the same while $\mathrm{Cu}$ foil was treated with $1 \mathrm{~min}$ acid (TFB) and 20 mins, respectively. Micro-Raman spectra of these dark regions reveal a higher D-band and G-band intensity at asimilar $\mathrm{G}^{\prime}$-band intensity. We conclude that the dark regions contain two types of carbon. The $\mathrm{G}^{\prime}$ band and part of the G-band originate from graphene, that is, growing from undamaged $\mathrm{Cu}$ regions and bridging the rough $\mathrm{Cu}$ areas. The D-band and the remainder of the Gband, however, come from defective graphene grown in the rough $\mathrm{Cu}$ areas. Consequently, the difference between Raman spectra obtained in the dark region and the pristine graphene exhibits a pronounced $\mathrm{D}$-band and a defect induced $\mathrm{D}^{\prime}$-band [15] akin to highly defective graphene (see inset Figure 4(c)).

\section{Conclusion}

In conclusion, we have analyzed the effect of catalyst surface morphology on the quality of graphene grown by CVD. We find that electropolishing can efficiently decrease the long wavelength roughness, or waviness, but at the expense of nanoparticle formation. These nanoparticles affect the probability of bilayer formation but have negligible effect on the electrical performance of the grown graphene. Increasing waviness, on the other hand, deteriorates the graphene quality and performance significantly as confirmed by etching experiments. Raman spectroscopy suggests that defective graphitic regions are forming in the high roughness areas 


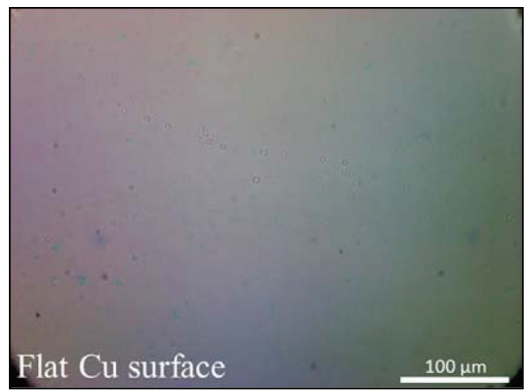

(a)

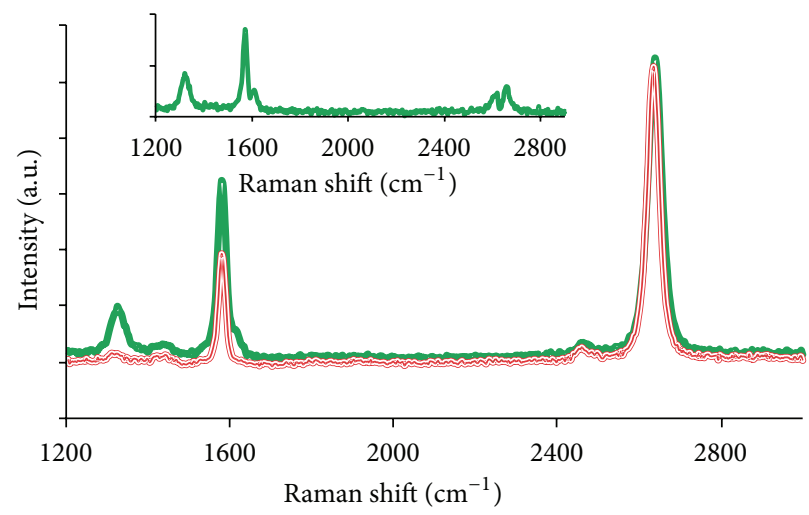

(c)

(b)

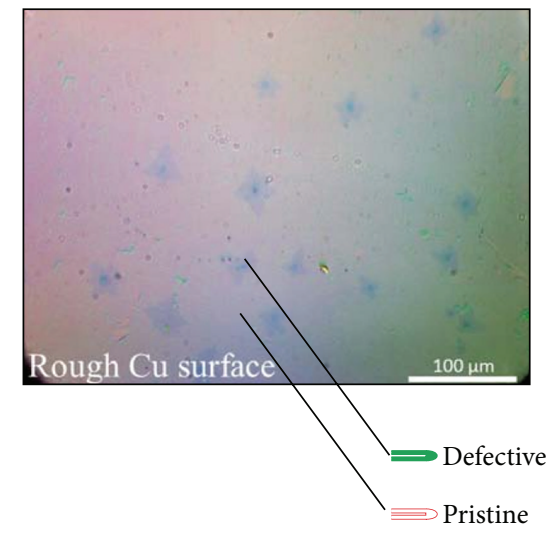

FIGURE 4: Graphene morphology upon acid etching. (a) and (b) Optical microscope images of graphene grown on (a) Cu foil with 1 min acid (TFB) treated and (b) 20 mins acid (TFB) treated Cu foil. (c) Raman spectra of dark and bright regions as indicated (inset: difference of spectra).

with detrimental effect on carrier mobility and sheet resistance.

These findings provide better insight into challenges for graphene formation but also suggest the limited importance of surface contaminants in the graphene formation process.

\section{Acknowledgments}

The authors acknowledge the financial support by the National Science Council, Taiwan, and the (Department of Industrial Technology) Ministry of Economic Affairs, Taiwan.

\section{References}

[1] F. Xia, T. Mueller, R. G. Mojarad et al., "Photocurrent imaging and efficient photon detection in a graphene transistor," Nano Letters, vol. 9, no. 3, pp. 1039-1044, 2009.

[2] X. Wang, L. Zhi, and K. Mullen, "Transparent, conductive graphene electrodes for dye-sensitized solar cells," Nano Letters, vol. 8, no. 1, pp. 323-327, 2008.

[3] X. Li, H. Zhu, K. Wang et al., "Graphene-on-silicon schottky junction solar cells," Advanced Materials, vol. 22, no. 25, pp. 2743-2748, 2010.
[4] S. L. Avila, K. M. Poulsen, R. Yakimova et al., "Non-Volatile photochemical gating of an epitaxial graphene/polymer heterostructure," Advanced Materials, vol. 23, no. 7, pp. 878-882, 2011.

[5] A. Reina, X. Jia, J. Ho et al., "Large area, few-layer graphene films on arbitrary substrates by chemical vapor deposition," Nano Letters, vol. 9, no. 1, pp. 30-35, 2009.

[6] L. Zhao, K. T. Rim, H. Zhou et al., "Influence of copper crystal surface on the CVD growth of large area monolayer graphene," Solid State Communications, vol. 151, no. 7, pp. 509-513, 2011.

[7] Z. Luo, Y. Lu, D. W. Singer et al., "Effect of substrate roughness and feedstock concentration on growth of wafer-scale graphene at atmospheric pressure," Chemistry of Materials, vol. 23, no. 6, pp. 1441-1447, 2011.

[8] Z. Yan, J. Lin, Z. Peng et al., "Toward the synthesis of wafer-scale single-crystal graphene on copper foils," American Chemical Society Nano, vol. 6, no. 10, pp. 9110-9117, 2012.

[9] H. Kim, C. Mattevi, M. R. Calvo et al., "Activation energy paths for graphene nucleation and growth on Cu," American Chemical Society Nano, vol. 6, no. 4, pp. 3614-3623, 2012.

[10] B. Zhang, W. H. Lee, R. Piner et al., "Low-temperature chemical vapor deposition growth of graphene from toluene on electropolished copper foils," American Chemical Society Nano, vol. 6, no. 3, pp. 2471-2476, 2012. 
[11] G. L. Wynick and C. J. Boehlert, "Use of electropolishing for enhanced metallic specimen preparation for electron backscatter diffraction analysis," Materials Characterization, vol. 55, no. 3, pp. 190-202, 2005.

[12] M. Hofmann, Y. C. Shin, Y. P. Hsieh, M. S. Dresselhaus, and J. Kong, "A facile tool for the characterization of two-dimensional materials grown by chemical vapor deposition," Nano Research, vol. 5, no. 7, pp. 504-511, 2012.

[13] A. W. Tsen, L. Brown, M. P. Levendorf et al., "Tailoring electrical transport across grain boundaries in polycrystalline graphene," Science, vol. 336, no. 6085, pp. 1143-1146, 2012.

[14] E. Loginova, N. C. Bartelt, P. J. Feibelmarr, and K. F. McCarty, "Factors influencing graphene growth on metal surfaces," New Journal of Physics, vol. 11, Article ID 063046, 2009.

[15] M. A. Pimenta, G. Dresselhaus, M. S. Dresselhaus, L. G. CanÇado, A. Jorio, and R. Saito, "Studying disorder in graphitebased systems by Raman spectroscopy," Physical Chemistry Chemical Physics, vol. 9, pp. 1276-1290, 2007. 

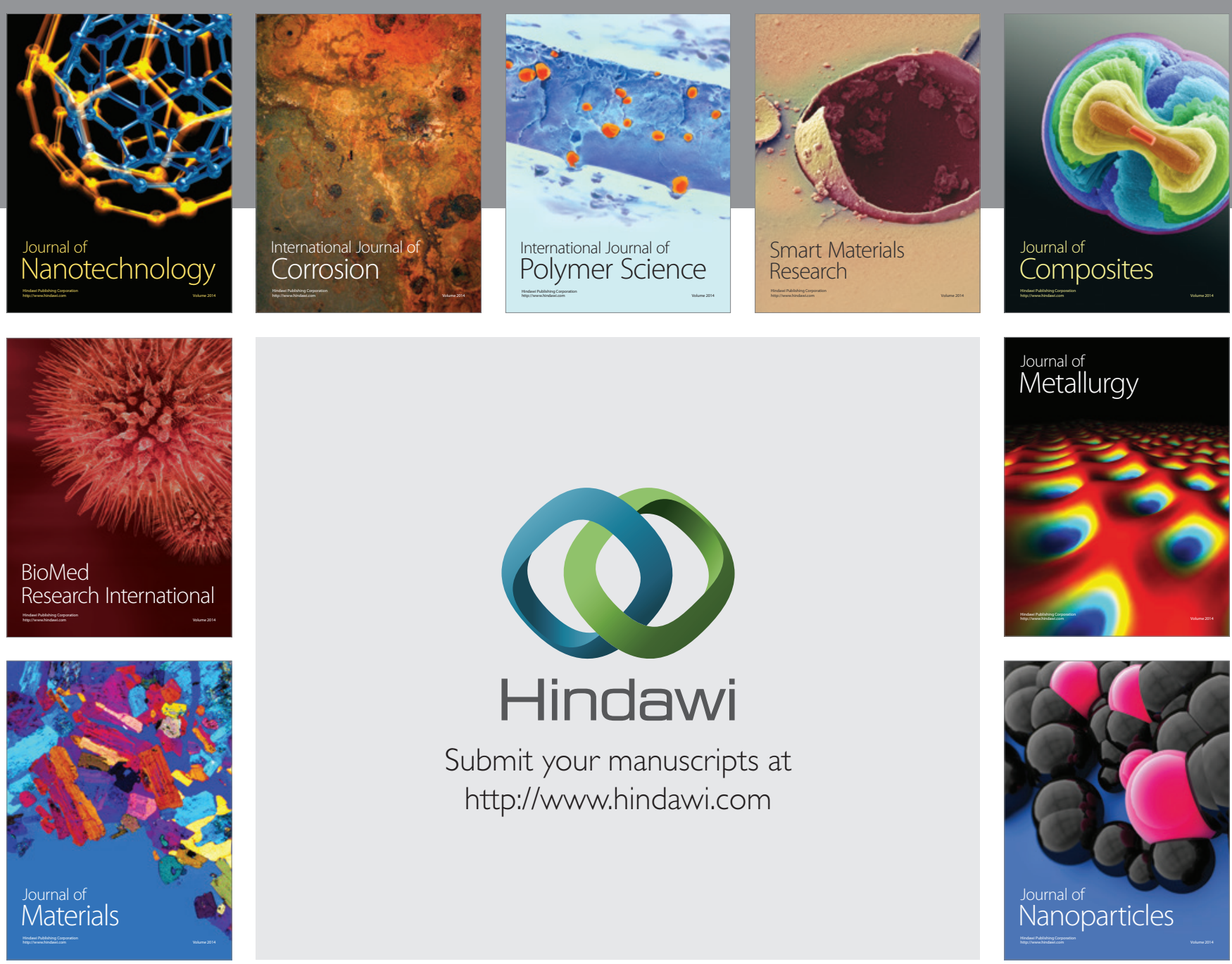

Submit your manuscripts at http://www.hindawi.com
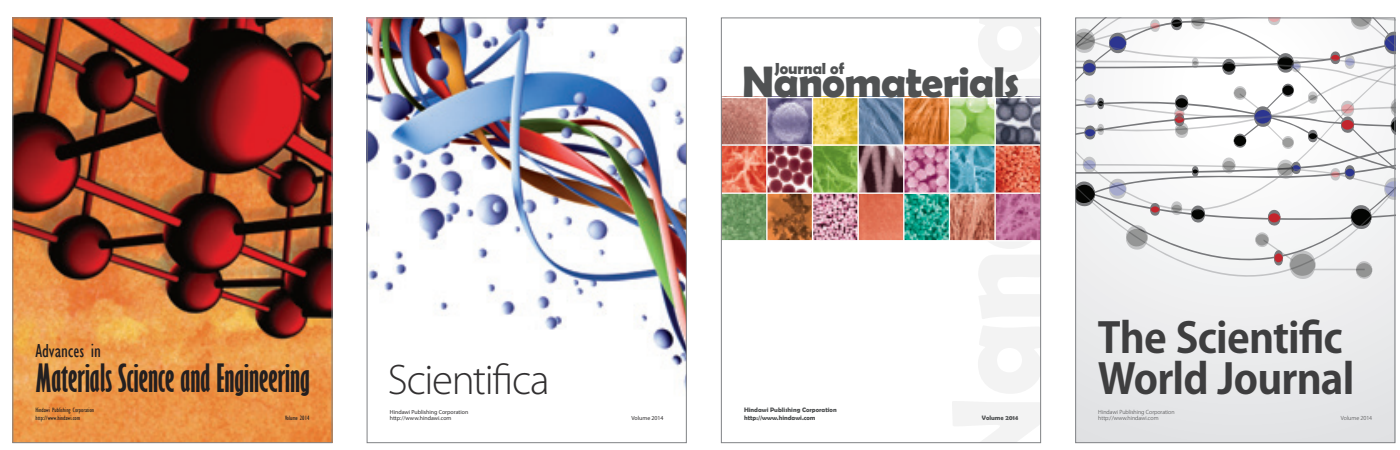

\section{The Scientific World Journal}
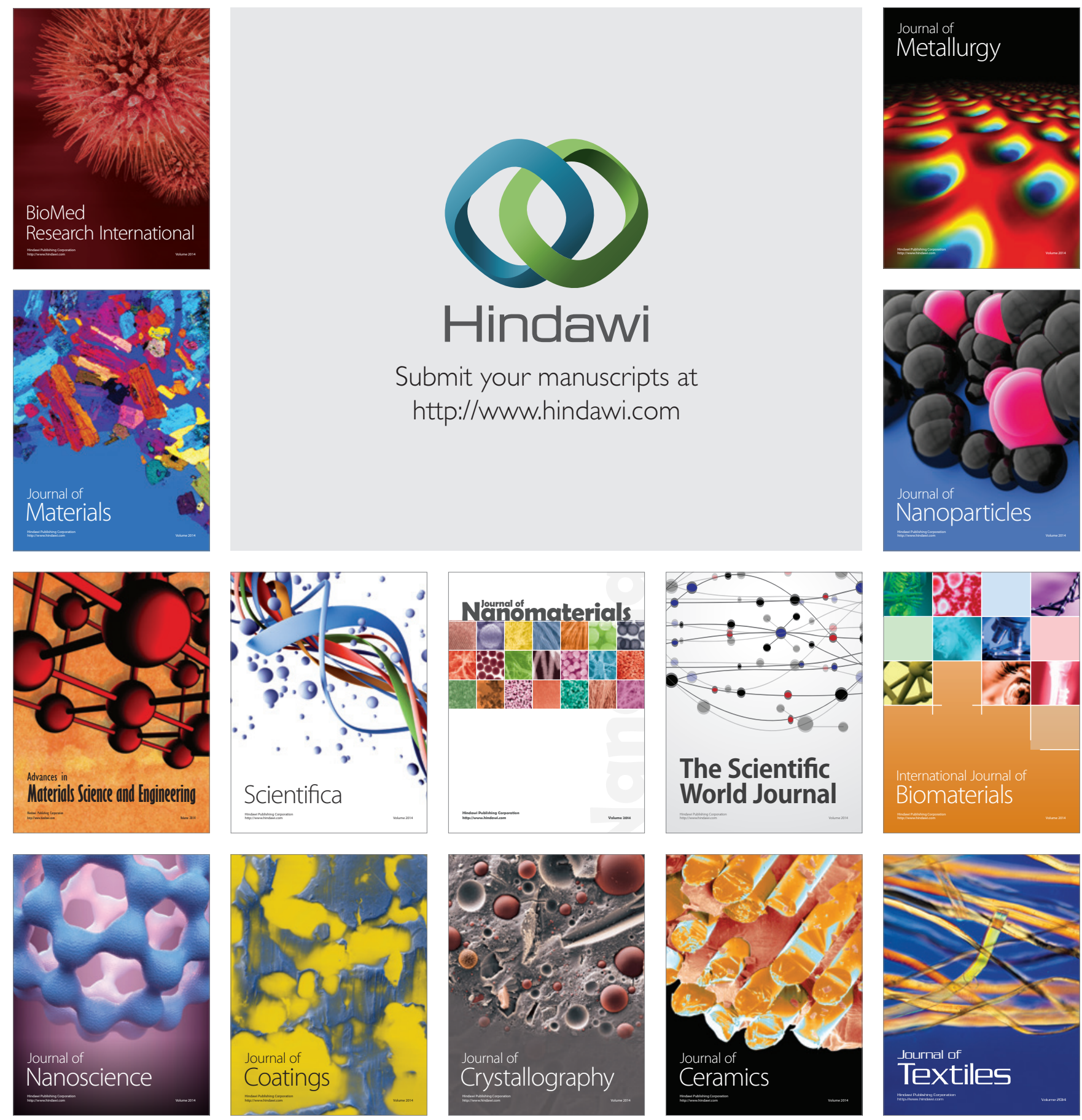